\title{
Tangence
}

\section{Référence littéraire et cliché critique : l'antonomase du nom d'auteur chez Berthelot Brunet}

\section{Élisabeth Nardout-Lafarge}

Numéro 44, juin 1994

La référence littéraire

URI : https://id.erudit.org/iderudit/025813ar

DOI : https://doi.org/10.7202/025813ar

Aller au sommaire du numéro

Éditeur(s)

Tangence

ISSN

0226-9554 (imprimé)

1710-0305 (numérique)

Découvrir la revue

Citer cet article

Nardout-Lafarge, É. (1994). Référence littéraire et cliché critique : l'antonomase du nom d'auteur chez Berthelot Brunet. Tangence, (44), 56-65.

https://doi.org/10.7202/025813ar d'utilisation que vous pouvez consulter en ligne.

https://apropos.erudit.org/fr/usagers/politique-dutilisation/ 


\section{Référence littéraire et cliché critique: l'antonomase du nom d'auteur chez Berthelot Brunet}

Élisabeth Nardout-Lafarge

Le discours critique ne saurait s'écrire sans le support (ou le secours) de la référence littéraire; alors que la critique savante a recours à la citation et à la note, la critique essayistique préfère la simple mention de noms d'auteurs pour esquisser son "paysage affiché"1. L'antonomase du nom d'auteur (le Baudelaire de son temps, "notre Rabelais") est l'une des modalités de la référence nominale ${ }^{2}$. Dupriez établit des degrés d'emploi et distingue les antonomases lexicalisées, répertoriées dans les dictionnaires, des antonomases discursives, ou "spontanées" dont la production est constante et potentiellement illimitée ${ }^{3}$. Élément mémoriel du lexique $^{4}$, l'antonomase est liée à la littérature comme l'attestent les Don Juan, Tartuffe, et autres Harpagon, le ton rabelaisien et le bovarysme.

La critique littéraire fait un large usage de cette figure et de ses dérivés qu'elle produit et reproduit, tant dans leur forme lexicalisée, puisque la plupart des noms d'auteurs ont donné lieu à la formation d'adjectifs (le roman balzacien, la phrase proustienne), que dans leur forme discursive où toutes les modalisations et toutes les enclosures sont possibles (une sorte de Queneau tragique, le nouveau Radiguet, etc.). Fondé sur la communisation d'un nom propre réel ou fictif (un Rastignac), ce procédé est étudié surtout par des linguistes pour qui il est l'un des cas intéressants

1 Antoine Compagnon, La seconde main ou le travail de la citation, Paris, Seuil, 1979, p. 333.

2 C'est le terme selon lequel Ruth Amossy définit la mention d'un nom d'auteur dans Les jeux de l'allusion littéraire dans Un beau ténébreux de Julien Gracq, Neuchâtel, Éditions de La Baconnière, coll. "Langages *, 1980, p. 29.

3 Bernard Dupriez, Antonomase , Gradus. Les procédés littéraires (Dictionnaire), Paris, U.G.E., 1984, p. 58-59.

4 Nelly Flaux, "L'antonomase du nom propre ou la mémoire du référent ", Langue française, $\mathrm{n}^{\circ} 92$, "Syntaxe et sémantique des noms propres *, décembre 1991 , p. $26-45$. 
de modification du nom propre ${ }^{5}$. Mais, en ce qu'elle fait apparaître l'axiologie sur laquelle se fonde le discours critique en dévoilant ses présupposés et ses visées rhétoriques, l'antonomase intéresse aussi l'histoire littéraire et l'analyse institutionnelle. $\grave{A}$ ce titre, les textes de Berthelot Brunet fournissent un corpus privilégié, à la fois parce que le procédé est chez lui étrangement systématisé et parce qu'il révèle la persistance d'une hiérarchie entre le comparant français et le comparé canadien-français.

Conteur, essayiste et critique littéraire, Berthelot Brunet (1901-1948) se définit lui-même comme un "polygraphe" dans son Histoire de la littérature canadienne-française, recueil de textes publié aux éditions de L'Arbre en $1946^{6}$, d'où sont tirés les exemples qui suivent. L'emploi même de l'antonomase suffirait à le confirmer, Brunet, malgré le titre de son ouvrage, ne se pose pas en historien de la littérature. Il jette sur l'ensemble du corpus canadien-français un regard résolument partial et impressionniste et l'usage des noms propres est peut-être justement l'un des supports de cette subjectivité.

Berthelot Brunet pratique une sorte de gradation dans l'usage de l'antonomase, de sa forme la plus simple: "La poésie patriotique du pays de Québec attend encore son Aragon" (p. 69), ou dans une construction symétrique: "Qui sait si Le Survenant n'est pas notre Grand Meaulnes?" (p. 130), "Au fond, François Hertel est surtout un chanoine Groulx badin" (p. 109), jusqu'à des structures plus complexes; ainsi parlant de Fréchette, qui, de l'avis même de Brunet, "se voulut le sosie et la doublure de Victor Hugo" (p. 63), il écrit: "[il] s'exila à Chicago, qui fut quelques années son île de Guernesey [...] De Chicago nous parvint [...] La voix d'un exilé qui sont ses Châtiments" (p. 64) et plus loin "Fréchette tenta à son tour son Hernani. (p. 66). Dans cet exemple, l'emploi antonomasique s'étend des titres d'œuvres aux épisodes de la biographie, tel l'exil évoqué par le toponyme l'île de Guernesey. La présentation de Fréchette (p. 63-67) est d'ailleurs entièrement soumise à ce parallèle avec Hugo. Brunet ne raconte de la vie politique et littéraire de

5 Voir notamment Marie-Noëlle Gary-Prieur, "Du Bach, du Colette : neutralisation du genre et recatégorisation des noms de personnes ", Le français moderne, 58, $\mathrm{n}^{\text {os }} 3-4$, p. 175-189.

6 Les numéros de pages renvoient à la réédition de 1970, due à Gilles Marcotte et André Major, Histoire de la littérature canadienne-française suivie de Portraits d'écrivains, Montréal, HMH, coll. "Reconnaissances", 1970. 
Fréchette que les épisodes qui peuvent trouver un équivalent dans la vie de l'écrivain français. Le réseau de correspondances ainsi mis en place souligne le mimétisme admiratif qui a guidé la carrière de Fréchette et renforce la supériorité du maître Hugo sur son épigone canadien-français.

La même figure permet également d'introduire des nuances. Ainsi Brunet dit de Louis-Hippolyte La Fontaine: "[il] fut d'abord le disciple de Papineau, [mais] se montre beaucoup moins emphatique. Disons que La Fontaine était le Poincaré de ce Briand. (p. 25). Ici, c'est le lecteur qui doit reconstituer l'antonomase double: Papineau est un Briand et La Fontaine un Poincaré, en même temps que le procédé est réinvesti de sens par la comparaison: La Fontaine est plus modéré que Papineau dans la même proportion et selon le même rapport que Poincaré vis-à-vis de Briand. On le voit, la systématisation du trait finit par menacer la lisibilité du texte; un incessant va-et-vient entre deux séries de noms propres littéraires se substitue au propos critique. Le texte de Brunet est saturé de variantes infinies de cette figure mais le procédé n'est pas nouveau dans la rhétorique du genre, ni dépassé non plus. Analysant la réception du roman québécois en France, Jacqueline Gérols cite quelques exemples de rapprochements littéraires très comparables à ceux qu'opéraient Brunet; ainsi, dans Le quotidien de Paris du 14 juillet 1976, Philippe Guilhon décrit Ducharme comme "Une sorte de Laforgue revu par Le canard encbaîné sur le chemin de la croix de Jarry "7.

Comme en témoignent de façon caricaturale les textes de Brunet, l'usage antonomasique des noms d'auteurs révèle d'abord une conception particulière de la littérature. En effet, charger le nom propre d'un contenu conceptuel ${ }^{8}$ n'est possible qu'à partir d'une typologie implicite dans laquelle les auteurs et les textes constituent des catégories, voire des programmes. Si "Le Survenant [est] notre Grand Meaulnes", c'est que Le grand Meaulnes, au-delà de l'œuvre elle-même, représente une valeur que le roman canadien-français doit atteindre. Dès lors, la littérature apparaît comme un système clos de modèles qui définissent des emplois à remplir (un Hugo, un Grand Meaulnes, un Aragon)

7 Jacqueline Gérols, Le roman québécois en France, La Salle, Hurtubise HMH, Cahiers du Québec, coll. "Littérature", 1984, p. 280.

8 Voir à ce sujet l'article de Bernard Meyer et Jean-Daniel Ballayn, "Autour de l'antonomase du nom propre", Poétique, n 46, avril 1981, p. 183-199. 
et du même coup, la description de l'évolution littéraire, tâche du critique ou de l'historien, se réduit à mesurer l'écart du nouveau par rapport à l'ancien. La récurrence des noms propres atteste l'importance dévolue ici aux individus et aux œuvres plutôt qu'aux idées et aux circonstances. Dans cette perspective, le nom propre soutient une vision essentialiste; l'antonomase postule en effet une essence des individus qui les dépasse puisqu'elle peut en définir d'autres.

Mais elle cristallise aussi le paradoxe de cette conception de l'art en inscrivant dans le nom propre à la fois l'exemplaire, donc l'unique (le génie, le chef-d'œuvre) et le multiple, la série, induits par le déterminant. L'emploi antonomasique marque donc une répétition - "notre Grand Meaulnes" sera forcément un nouveau Grand Meaulnes - et dans le même temps la met en doute on ne peut pas reproduire du propre. Selon Nelly Flaux, "c'est [la] résistance du Nom propre au statut de Nom commun [qui] soutient la figure de l'antonomase " 9 . La persistance du propre dans la communisation, ou, pour le dire autrement, du référent d'origine dans le nouvel emploi, fait également de l'antonomase une figure très efficace de la dévaluation. C'est en tout cas ce que donnent à lire certaines enclosures typiques : “ un Picasso de banlieue ", "un Rastignac de village" marquent non seulement le décalage avec le modèle, mais la caricature ou la pose, et les noms propres renvoient moins à ce que sont les sujets ainsi décrits qu'à ce pour quoi ils se prennent. C'est bien d'ailleurs par le maintien d'une distance que Brunet sanctionne finalement le parallèle entre Fréchette et Hugo, en annulant l'antonomase par une autre modification du nom propre, figure finale puisque tautologique: "Seul Victor Hugo pouvait faire du Victor Hugo" (p. 66).

Dans Histoire de la littérature canadienne-française, l'ambiguité du procédé, qui pose une équivalence pour en rappeller aussitôt l'impossibilité, souligne l'ambivalence du discours de Brunet sur la littérature française. En effet, la quasi-totalité des comparants auxquels il a recours renvoient à des textes ou des auteurs français. Ces noms propres, et c'est là leur premier intérêt pour l'histoire littéraire, renseignent sur ce que devait être la culture littéraire d'un intellectuel canadien-français des années quarante, à quelques variantes près, dues à l'originalité de

9 Nelly Flaux, op. cit., p. 37. 
60

Brunet, anticonformiste, curieux et libre penseur. Mais dans ces textes, la littérature, l'histoire littéraire, culturelle et politique françaises constituent plus qu'un bagage encyclopédique commun au critique et aux lecteurs, c'est un relais indispensable à l'appréhension des textes canadiens-français. Brunet ne peut traiter la littérature canadienne-française que par rapport et au moyen de la littérature française, érigée en mesure et en critère de l'existence littéraire. C'est toujours face aux textes français que sont définis les textes canadiens-français, dans une comparaison qui n'est plus simplement un artifice du discours critique mais un de ses fondements. Brunet se livre à une sévère autocritique, aussi la production canadienne-française est-elle presque toujours jugée inférieure à la production française, dans de très rares cas, égale. Équivalence de qualité dont la reconnaissance emprunte d'ailleurs une formulation significative: "Lorsque [Bonbeur d'occasion] parut, l'étonnement se répandit partout: cette femme était aussi vraie que les bons réalistes de France ou de l'étranger" (p. 131).

Le rôle d'autorité absolue dévolu aux textes français et, son corollaire, l'infériorisation systématique des textes canadiensfrançais ne sont pas spécifiques à Berthelot Brunet; ils s'inscrivent dans une longue tradition critique issue du XIx ${ }^{\mathrm{e}}$ siècle. MarieAndrée Beaudet rappelle, dans Langue et littérature au Québec. 1895-1914, l'affirmation de Jules Fournier dans La revue canadienne de juillet 1906: "Il n'y a pas de littérature canadiennefrançaise. La chose ne se discute pas ${ }^{10}$. Longtemps encore après Brunet, les textes français resteront, plus ou moins implicitement, la norme et la mesure de la littérature. La fixité de la construction antonomasique, qui maintient l'élément français en position de comparant et l'élément canadien-français en position de comparé, est d'autant plus significative que Brunet tient, notamment dans Histoire de la littérature française ${ }^{11}$, un discours volontiers iconoclaste sur les textes français. Ici, au contraire, tous les itinéraires littéraires qu'évoque Brunet finissent par être réductibles à des modèles français préexistants et son propos se limite à établir d'infinis parallèles, à évaluer des degrés de ressemblance. Toute

10 Marie-Andrée Beaudet, Langue et littérature au Québec. 1895-1914. L'impact de la question linguistique sur la formation du cbamp littéraire, Montréal, l'Hexagone; coll. "Essais littéraires", 1991, p. 62.

11 Berthelot Brunet, Histoire de la litterature française, Montréal, $\mathrm{HMH}$, coll. "Reconnaissances ", 1970. 
description du corpus canadien-français se trouve évacuée et la transposition s'avère finalement une stratégie d'évitement.

Cette position de calque de la littérature française assignée à la littérature canadienne-française est souvent signifiée par l'emploi du possessif en position de déterminant. Ainsi, la phrase "[L']Histoire [de Garneau], ce sont nos sagas et notre Chanson de Roland" (p. 32) postule que la littérature canadienne-française doit commencer comme la littérature française et produire un équivalent à La chanson de Roland. L'usage du possessif devant un nom littéraire français peut marquer le manque, par exemple dans "la poésie patriotique attend [...] son Aragon" où le nom emprunté ailleurs désigne la place vacante; il peut signaler également l'appropriation, souhaitée mais incertaine dans "Nos SainteBeuves, nos Taines, nos Thibaudets et nos Brémonds" (p. 168), - où l'on notera la lexicalisation par l'emploi du pluriel - ou réalisée dans "l'Histoire de Garneau [c'est] notre Chanson de Roland. Pourtant l'antonomase, contrairement à une expression synonymique telle "notre épopée fondatrice" inscrit simultanément le même et la différence; le possessif "notre" ne signifie pas que l'Histoire de Garneau est La chanson de Roland mais qu'elle en tient lieu, comme une doublure pour l'acteur, une copie pour l'original; le déterminant est donc, dans cet emploi métaphorique, le support d'une minorisation. De plus, comme marque d'appropriation, le possessif ouvre toujours sur la fragmentation et la multiplicité du référent d'origine; ainsi, le syntagme "Notre Rabelais", employé de manière autonome, présuppose l'existence possible de plusieurs Rabelais, dont l'un est nôtre ; l'antonomase réalise beaucoup plus complètement l'appropriation que n'aurait pu le faire l'expression "notre lecture de Rabelais", plus respectueuse de l'unicité du référent d'origine.

L'antonomase du nom d'auteur pose aussi un problème de décodage. En effet, le contenu conceptuel attribué au nom propre ou au titre doit être déchiffrable. Dans le langage courant, l'efficacité de l'antonomase repose sur la possibilité d'associer au nom propre un ensemble de prédicats qui lui sont liés (l'avarice à Harpagon, l'ambition à Rastignac), possibilité que garantit la compétence culturelle dans le cas des antonomases lexicalisées, et que le contexte assure pour les antonomases discursives. Dans le discours critique, la fortune du trait tient d'abord à son pouvoir de classement, dans des séries littéraires déjà existantes - qu'il s'agisse d'un ton, d'une école ou d'un genre - et sur une échelle 
62

de valeurs - par l'effet légitimant qu'a le nom comparant sur le nom comparé. L'antonomase du nom d'auteur classe et, dans les textes de Brunet, il faut l'entendre au pied de la lettre: elle assigne des places.

Si le classement est aisément décodable en tant que jugement de valeur, la fonction de description de l'antonomase joue sur l'ambiguilté en laissant le lecteur libre des associations qu'appelle pour lui le nom d'un écrivain ou le titre d'un texte. Dans ce système d'analogie, le concept que le nom propre a charge d'incarner ne peut pas être clair et le consensus sur lequel l'antonomase prétend s'appuyer reste flou. Le nom propre recouvre en effet une incertitude en ce qu'il englobe à la fois le référent d'origine (l'auteur, le texte cités) mais aussi l'ensemble des jugements critiques qui en ont déterminé l'essence et coté la valeur. L'antonomase, comme tous les clichés, est un raccourci. Elle utilise sans les énoncer ces jugements critiques et les fond au nom par une opération qui les réitère et interdit leur questionnement; dans ce sens, elle scelle le pacte entre nom et renom. Ainsi de quelle interprétation du Grand Meaulnes Brunet rapproche-t-il Le Survenant? Le roman du rêve, de l'adolescence ou l'idée plus générale d'un succès unique?

Enfin, l'emploi de l'antonomase permet à Brunet de faire jouer un nom propre sans dévoiler la valeur que lui-même y attache. Dans l'exemple "La poésie patriotique [...] attend [...] son Aragon", Aragon réfère à la fois à l'écrivain de ce nom, à son œuvre, à la valeur institutionnelle qui lui est attribuée en 1945 et à l'opinion particulière que Brunet s'en fait. Le recours au contexte ne lève pas l'ambiguité :

Du reste Chapman aura peut-être été l'un des derniers de nos poètes patriotes à l'excès, voire chauvins: après lui, on écrira des poèmes nationaux, mais on ne recommencera plus guère l'ô Canada. La poésie patriotique du pays de Québec attend encore son Aragon (p. 69).

Que signifie ici le nom d'Aragon? Faut-il comprendre une qualité de texte ou une attitude de poète? Question d'autant plus ambiguë que Brunet dénonce, dans les pages de La nouvelle relève, un Aragon surréaliste, communiste et anti-patriote. L'usage d'une autre antonomase, qui consiste à désigner la poésie patriotique de Chapman par le titre de l'hymne canadien dont l'auteur est Adolphe-Basile Routhier, complique encore l'interprétation de cet 
énoncé. Alors qu'on peut être tenté, à la première lecture, de substituer à "son Aragon", une définition du type "son grand poète" ou "sa réussite", une lecture plus attentive et mieux informée des positions de Brunet pourrait ajouter aux prédicats que recouvre le nom d'Aragon "poète officiel, dogmatisme politique et littéraire, poésie grandiloquente". Ainsi compris, le procédé se renverserait; le comparant serait canadien-français (l'ô Canada) et le comparé français (les textes patriotiques d'Aragon), inversion d'ailleurs partielle puisque c'est encore l'absence de valeur qu'incarne le terme canadien-français. Cette interprétation semble démentie par la tournure "attend[re] encore" qui introduit conventionnellement un élément positif. Le nom "Aragon" conserve donc une certaine indiscernabilité. Il semble par conséquent que la pluralité des interprétations possibles fasse partie de l'effet recherché par l'antonomase.

En tant que référence littéraire, l'antonomase est une marque intertextuelle, la plus rudimentaire peut-être puisqu'elle se limite à un nom, un adjectif dérivé ou un titre, qui fonctionnent, de manière métonymique, comme appel et renvoi à des textes et des savoirs ${ }^{12}$. Comme les autres manifestations intertextuelles, l'antonomase délimite et signe un territoire littéraire. Les noms d'auteurs, de personnages, les titres sont autant de balises qui fixent une compétence et tentent de la cautionner. Le procédé est plus nécessaire encore pour le type d'essai littéraire que pratique Brunet, genre qui doit assumer l'arbitraire de ses jugements et la subjectivité de ses critères. L'antonomase du nom d'auteur n'est alors que la manifestation la plus transparente de l'autolégitimation que produit le discours critique; à ce titre, elle incarne, jusqu'à l'utopie, son autonomisation: comment imaginer en effet des catégories plus spécifiquement littéraires que celles qui sont formées à partir des noms d'écrivains?

Il n'est pas inutile de rappeler à cet égard à quel point cette figure, qui investit du sens figuré dans un nom propre, contribue à la constitution des idiolectes ou des micro-langages par lesquels se distinguent et se resserrent les groupes. Nelly Flaux cite à ce propos l'une des constructions antonomasiques dont fourmille $\mathrm{La}$

12 J'ai tenté ailleurs de préciser le rapport que la référence littéraire entretient avec l'intertexte: "La mise en scène textuelle de la référence littéraire chez Hertel et Lemelin *, Études françaises, vol XXIX, no 1, "Bibliothèques imaginaires du roman québécois ", printemps 1993, p. 77-94. 
64

recherche du temps perdu, "c'est de l'Oriane tout pur"13. De même, l'usage de cette figure constitue, dans les textes de Brunet, un pacte de lecture, établi sur le mode de la connivence. Les antonomases enchâssées, filées comme on peut le dire des métaphores, ont aussi pour fonction de déterminer des lecteurs aux compétences littéraires suffisantes pour décrypter les allusions. Un tel système aura donc différents degrés d'ouverture ou d'hermétisme; ainsi, comme le montre l'exemple de Proust, on peut référer à cet écrivain sans mentionner son nom ni les titres de son œuvre, grâce à d'autres termes qui, pour les lecteurs assidus, fonctionnent comme référence: la madeleine, "faire catleya ", La vue de Delf, etc.

L'antonomase est aussi un commerce de noms propres; dans la rhétorique de Berthelot Brunet, l'usage abusif de l'auctoritas indique en creux l'illégitimité d'une parole et donc de son propre nom:

Le critique est - qui ne l'a dit? - le parent pauvre de la littérature. Il en est aussi le jeune arriviste qui n'a su où trouver encore où se faufiler et qui, à parler de soleils plus ou moins brillants, veut attirer sur lui les rayons, plus ou moins lumineux [...] je parle de la critique, genre roturier, et j'en veux parler en critique. Le critique est le plus souvent, dans ses commencements, celui qui, sous couleur de faire le portrait d'un autre, veut attirer l'eau à son moulin (p. 167).

On ne saurait définir plus clairement le processus d'anoblissement auquel se livre Brunet en parsemant son propre discours de noms littéraires, français et prestigieux, ainsi que l'enjeu légitimant d'une telle pratique.

Pourtant, la décrivant, Brunet s'en distance et s'en protège; la surenchère des noms propres, convoquée pour soutenir et conforter le sien, est finalement dissoute dans l'ironie: "Nous avons trop de critiques, je l'accorde: je donnerais dix Choquette pour un demi-Fournier, dix P. de M. pour un Parizeau et deux Desrochers et trois quarts pour un Valdombre" (p. 173). À l'antonomase s'ajoute ici la comptabilisation qui achève la communisation en ce qu'elle nie, aussi radicalement que possible, le propre du nom. La position de Brunet est peut-être tout entière dans cette ambiguïté, qui est aussi celle qui sous-tend la figure: l'emploi antonoma-

13 Nelly Flaux, op. cit., p. 43. 
sique désacralise les noms propres puisqu'il en fait des noms communs, donc des objets éventuellement comptables et échangeables. Mais le propre fait retour et l'antonomase reconduit la consécration du nom qu'elle cite, d'autant que cet emploi du nom est l'une des marques de la gloire littéraire. On ne s'étonnera donc plus que Brunet s'enlise dans la répétition de ce procédé alors même qu'il travaille justement à une entreprise de consécration de la littérature canadienne-française. Bien qu'il lui soit indispensable, le recours aux textes français fait écran à son analyse. L'antonomase du nom d'auteur est le lieu où se nouent ces contradictions, une sorte de scène sur laquelle Brunet piétinerait en vain ses fétiches. 